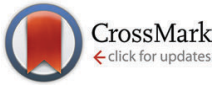

Cite this: Chem. Commun., 2015, 51,4880

Received 19th January 2015, Accepted 10th February 2015

DOI: $10.1039 / \mathrm{c5cc00530b}$

www.rsc.org/chemcomm

\title{
Polymerase synthesis of DNA labelled with benzylidene cyanoacetamide-based fluorescent molecular rotors: fluorescent light-up probes for DNA-binding proteins $\dagger$
}

\author{
Dmytro Dziuba, ${ }^{a}$ Radek Pohl ${ }^{a}$ and Michal Hocek*ab
}

\begin{abstract}
Viscosity-sensitive fluorophores, fluorescent molecular rotors based on aminobenzylidene-cyanoacetamide moiety, were tethered to 2 '-deoxycytidine triphosphate via a propargylamine linker and incorporated into DNA by polymerases in primer extension, nicking enzyme amplification or PCR. DNA probes incorporating modified nucleosides show a light-up response upon binding to a protein.
\end{abstract}

Fluorescent probes targeting DNA-binding proteins are of great value for cell biology to study signaling pathways, for medicinal chemistry to develop drug screening assays, and for clinical chemistry to detect biomarkers. ${ }^{1}$ Although fluorescence spectroscopy is a powerful method for studying biomolecular interactions, the existing methods of detection of DNA-protein binding in solutions using fluorescent reporting groups suffer several limitations. Most of the current DNAbased fluorescent probes are designed for the detection of proteins exhibiting enzymatic activity. ${ }^{1 a}$ Examples are nuclease ${ }^{2}$ and uracil DNA glycosylase ${ }^{3}$ probes based on artificial emissive nucleoside analogues quenched by stacking interactions with neighbouring nucleobases within the double helix. In contrast to enzymes which modify the chemical structure of DNA, many biologically important DNA-binding proteins, i.e. transcription factors or histones, do not alter the chemical composition of DNA and thus some more sophisticated approaches are required for their detection. Several methods based on molecular beacons ${ }^{1 b, 4}$ and environmentally sensitive fluorescent nucleoside analogues ${ }^{5}$ have been developed for this purpose. These methods usually require de novo synthesis of the probe for each particular target via solid phase phosphoramidite synthesis, which make them cost-consuming and can limit high-throughput applications. In this respect, polymerase construction ${ }^{6}$ of DNA-based probes using chemically modified deoxynucleoside triphosphates (dNTPs)

\footnotetext{
${ }^{a}$ Institute of Organic Chemistry and Biochemistry, Academy of Sciences of the Czech Republic, Gilead \& IOCB Research Center, Flemingovo nam. 2, CZ-16610 Prague 6, Czech Republic. E-mail: hocek@uochb.cas.cz

${ }^{b}$ Department of Organic Chemistry, Faculty of Science, Charles University in Prague, Hlavova 8, CZ-12843 Prague 2, Czech Republic

$\dagger$ Electronic supplementary information (ESI) available: Additional figures and tables, experimental details, copies of NMR and MALDI spectra. See DOI: 10.1039/ c5cc00530b
}

would be advantageous. In our proof-of-the-principle studies we showed that dNTPs bearing suitable environmentally sensitive fluorescent reporter (either solvatochromic aminophthalimide ${ }^{7}$ or GFP-fluorophore as a molecular rotor $^{8}$ ) can be incorporated into DNA by polymerases. The resulting probes showed a light-up response upon binding to proteins, although the increase of fluorescence was rather low (max. 2.5-fold). ${ }^{7,8}$ Therefore, development of more sensitive fluorophores is highly desirable.

Here we report improved fluorescent dNTPs based on molecular rotors, their enzymatic incorporation into DNA and use for sensing of DNA-protein interactions. Fluorescent molecular rotors (FMRs) are a class of fluorescent dyes sensitive to local viscosity. The most widely used are $p$-( $N, N$-dialkylamino)benzylidene-malononitriles DCVJ and CCVJ (1a, 2, Fig. 1a). ${ }^{9,10,14}$ These FMRs show increased fluorescence intensity in media of high viscosity (Fig. 1b). They found applications in life sciences as fluorescent viscosity probes for microheterogeneous systems, such as cytoplasm and cell membranes, ${ }^{9}$ but the possibility to probe biomolecular interactions by emission of FMRs is only poorly explored. ${ }^{10}$ In the field of DNA studies, FMRs were used to study pre-melting of $\mathrm{DNA}^{11}$ and probing of G-quadruplexes. ${ }^{12}$ Several emissive nucleoside analogues have been shown to be sensitive to the viscosity of the media, ${ }^{13}$ but none of them was used for the probing of interactions with proteins. In this study we hypothesized that a (a)

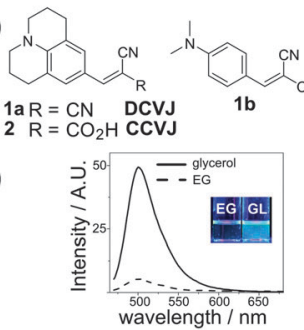

(c)

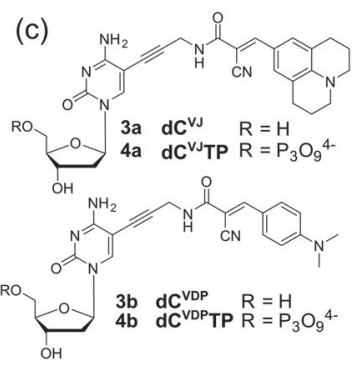

Fig. 1 (a) Structure of fluorescent molecular rotors DCVJ and CCVJ. (b) Uncorrected fluorescence spectra $\left(\lambda_{\text {ex }}=460 \mathrm{~nm}\right.$ ) of DCVJ in ethylene glycol and glycerol; inset - photography of the solutions of DCVJ in ethylene glycol (left) and glycerol (right) under a UV lamp. (c) Structures of FMR-labelled nucleosides $(\mathbf{3} \mathbf{a}-\mathbf{b})$ and triphosphates $(\mathbf{4} \mathbf{a}-\mathbf{b})$ described in this work. 
nucleoside analogue bearing a CCVJ-type FMR attached to a nucleobase via a short linker will be a useful probe for protein binding. CCVJ is known to increase fluorescence upon binding with tight hydrophobic sites of proteins, such as albumins or antibodies. ${ }^{14}$ Although DNA binding proteins usually do not have hydrophobic pockets, the layer of water molecules next to the protein interface (the hydration shell) exhibits retarded molecular dynamics comparing to water in the bulk. ${ }^{15}$ This might provide a rational basis for protein-induced fluorescence enhancement (PIFE). ${ }^{16}$

Our design of FMR-labelled nucleosides is shown in Fig. 1c. We use a short propargylic linker to connect the $p$-( $N, N$-dialkylamino)benzylidene-malononitrile to position 5 of cytosine. Two distinct $N, N$-dialkylamino-aryl groups were used to give a julolidine derivative $\left(\mathbf{d C}^{\mathbf{V J}}\right)$ and its less bulky $N, N$-dimethylaniline analogue $\left(\mathbf{d C}^{\mathbf{V D P}}\right)$. The FMR-nucleosides were synthesized using a modular strategy based on the Sonogashira coupling of 5-iodo-dC with the corresponding fluorophore-linked acetylene (Scheme S1 in the ESI $\dagger$ ).

Having in hands the nucleosides we compared their photophysical and viscosity-sensitive properties with reference compounds 1a, $\mathbf{b}^{17}$ (Fig. 2, Table 1). The introduction of a nucleoside moiety blue-shifted the absorption spectra, whereas the positions of emission maxima were not affected. The sensitivity to viscosity was measured using the Forster-Hoffmann theory, ${ }^{9 a, 17}$ stating that the logarithm of fluorescence intensity $(F)$ depends on the logarithm of the viscosity of the media $(\eta)$ as follows:

$$
\log F=x \log \eta+C
$$

here $x$ is the viscosity sensitivity which is an intrinsic characteristic of a molecular rotor. According to the theoretical predictions, the maximum value of $x$ is 0.66 , whereas, for the vast majority of FMRs, the $x$ value ranges from 0.4 to $0.6 .{ }^{17}$ The sensitivity to viscosity was slightly higher for the nucleosides compared to the reference, whereas this improvement was more significant in the cases of $\mathbf{1 b}$ and $\mathbf{3 b}$ (Fig. 2). Notably, the values of $x$ for nucleosides
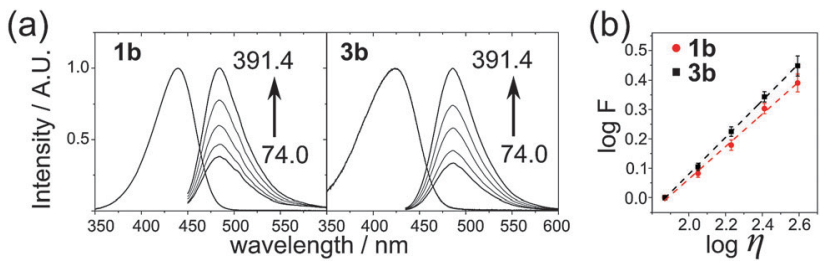

Fig. 2 The improvement of viscosity-sensitive properties of FMR-nucleoside 3b compared to $\mathbf{1 b}$. (a) Absorption (EG) and uncorrected fluorescence (EGglycerol) spectra of $\mathbf{1} \mathbf{b}$ and $\mathbf{3} \mathbf{b}$; the viscosity of samples was (from bottom to top): $74.0,112.2,170.2,258.1$ and $391.4 \mathrm{mPa}$ s. (b) The Forster-Hoffmann plot

Table 1 Photophysical properties of fluorescent molecular rotors

\begin{tabular}{llll}
\hline Compound & $\lambda_{\mathrm{abs}}{ }^{a}$ & $\lambda_{\mathrm{em}}{ }^{b}$ & $x^{c}$ \\
\hline $\mathbf{1 a}$ & 466 & 503 & $0.594 \pm 0.028$ \\
$\mathbf{1 b}$ & 440 & 486 & $0.554 \pm 0.020$ \\
$\mathbf{3 a}\left(\mathbf{d C}^{\mathbf{V J}}\right)$ & 452 & 506 & $0.630 \pm 0.021$ \\
$\mathbf{3 b}\left(\mathbf{d C}^{\mathbf{V D P}}\right)$ & 423 & 488 & $0.627 \pm 0.022$
\end{tabular}

${ }^{a}$ Position in nm of the maximum of the absorption in EG. ${ }^{b}$ Position in $\mathrm{nm}$ of the maximum of the emission in EG-glycerol. ${ }^{c}$ Viscosity sensitivity obtained from the Forster-Hoffmann equation. (a)
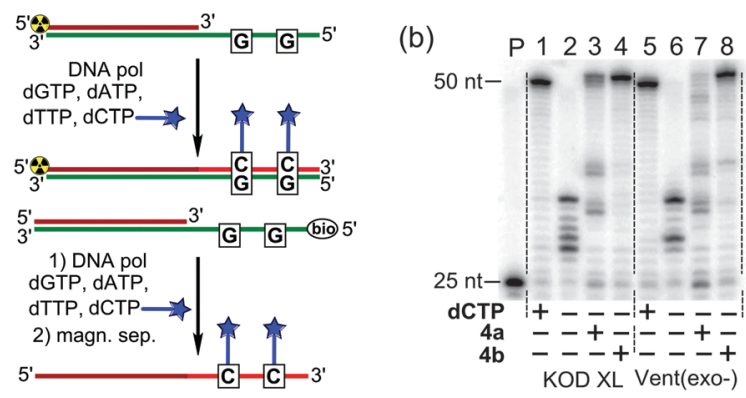

Fig. 3 (a) The scheme of primer extension (PEX) with FMR-modified $\mathbf{d C}^{\mathrm{X}}$ TPs; (b) PAGE analysis of PEX with template T1X and primer P1X performed by KOD XL (lanes 1-4) and Vent(exo-) (lanes 5-8) polymerases; and (c) preparation of labelled ssDNA by PEX followed by magnetic separation.

$3 \mathbf{a}$ and $3 \mathbf{b}$ ( 0.630 and 0.627 , respectively) are pretty close to the theoretical limit (0.66) indicating that title compounds exhibit highly favourable sensing properties.

Then we proceeded to the synthesis of DNA. For the enzymatic incorporation, nucleosides $\mathbf{3 a}$ and $\mathbf{3} \mathbf{b}$ were phosphorylated at $5^{\prime}$ using the procedure of $\mathrm{Ludwig}^{18}$ to give corresponding triphosphates 4a and 4b (Fig. 1c; Scheme S1, ESI $\dagger$ ). We examined the possibility of incorporating the modified dNTPs into DNA by enzymatic methods, i.e. primer extension (PEX), nicking enzyme amplification reaction (NEAR) and polymerase chain reaction (PCR). ${ }^{6 c, 19}$

At first we tested the modified $\mathbf{d C}^{\mathbf{X}^{\mathbf{T}}} \mathbf{T P s}$ in combination with three natural dNTPs in PEX with KOD XL and Vent(exo-) DNA polymerases (Fig. 3a). PAGE analysis of the PEX reaction (Fig. 3b) shows that $\mathbf{d C}^{\mathbf{V D P}} \mathbf{T P}$ was a good substrate for both enzymes tested (lanes 4 and 8) and gave full-length extended products, whereas the bulky $\mathbf{d C}^{\mathbf{V}} \mathbf{T} \mathbf{P}$ was less efficient, giving a number of shorter products (lanes 3 and 7). Nucleotide $\mathbf{d C}^{\text {VDP }}$ was nicely incorporated into DNA even at a high density (Fig. S1, ESI $\dagger$ ). Single-stranded oligonucleotides (ssONs) were prepared by PEX using a biotinylated template and isolated by magnetoseparation with streptavidine-coated magnetic beads (Fig. 3c). MALDI-TOF analysis of the ssON containing modified nucleotides $\mathbf{d C}^{\mathbf{x}}$ confirmed the correct full-length products (Fig. S1 in the ESI $\dagger$ ) and UV-vis spectroscopy confirmed the absence of non-specific binding of $\mathbf{d C}^{\mathbf{V D P}} \mathbf{T P}$ to DNA (Fig. S2, ESI $\dagger$ ).

Then, we tested the modified triphosphates in the nicking enzyme amplification reaction (NEAR), ${ }^{19 b, c}$ a two-step linear isothermal amplification process for synthesis of short ssONs (Fig. 4a). The results of NEAR with different templates (12-mer ON, containing one or three $\mathrm{dC}^{\mathrm{x}}$ modifications) are shown in Fig. 4b. The NEAR works well with both modified $\mathbf{d C}^{\mathbf{X}} \mathbf{T P s}$, but the yield was higher in the case of less dense (a)

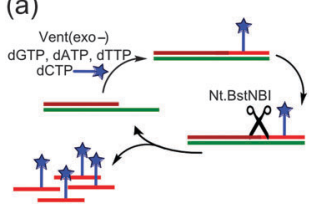

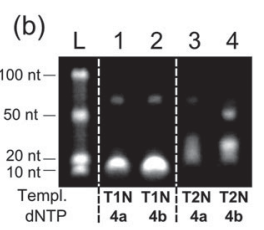

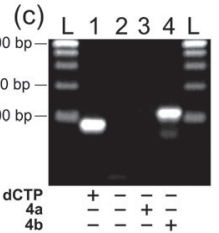

Fig. 4 Enzymatic synthesis of FMR-modified DNA by NEAR and PCR. (a) Schematic representation of NEAR; (b) agarose gel analysis of NEAR products; and (c) agarose gel analysis of PCR products. 
labelling. Correct incorporation of modified dNTPs was also confirmed by MALDI-TOF spectrometry. The NEAR on a semipreparative scale was also performed using $\mathbf{d C}^{\mathbf{V D P}} \mathbf{T P}$ followed by HPLC purification, yielding the corresponding 12-mer modified oligonucleotide in $1.9 \mathrm{nmol}$ yield $(0.5 \mathrm{~mL}$ scale). To further explore the applicability of the FMR-modified dNTPs, we tested them in PCR. A series of optimization experiments have shown that $\mathbf{d C}^{\mathbf{V D P}}$ (but not $\mathbf{d C}^{\mathbf{V J}}$ ) can be sufficiently incorporated by KOD XL DNA polymerase using $\mathbf{d C}^{\mathbf{V D P}} \mathbf{T P}$ as the substrate (Fig. 4c), although it requires higher concentration of $\mathbf{d C}^{\mathbf{V D P}} \mathbf{T P}$, increased number of cycles and longer elongation time. Altogether, these results indicate that $\mathbf{d C}^{\mathbf{V D P}} \mathbf{T P}$ is a good substrate for enzymatic synthesis of DNA using PEX, NEAR and PCR.

Finally, we examined the usability of fluorescent molecular rotors attached to DNA as reporting groups for protein-DNA interactions. Binding studies in solution were performed using a 30-mer ssDNA probe ON1 (Table S2 in the ESI $\dagger$ ) obtained by PEX with magnetoseparation (Fig. 3c) and single-strand binding protein from $E$. coli (SSB), exhibiting a non-sequence specific binding to SSDNA. $^{20} \mathrm{We}$ observed a significant 4-fold increase of fluorescence upon titration of DNA by SSB with a stoichiometry of interaction $2: 1$ (Fig. 5). Since at least $56 \mathrm{nt}$ ssDNA is needed to wrap around the SSB tetramer under the conditions used, ${ }^{21}$ the short ONs used in our study apparently bind in a higher ratio. Notably, the maximal increase of fluorescence was higher than with our previous probes. ${ }^{7,8}$

To rule out possible non-specific interaction between the DNAtethered fluorophores and protein, we also studied the influence of bovine serum albumin (BSA), which does not bind to DNA but possess hydrophobic pockets where FMR can bind. ${ }^{14 b}$ No fluorescence enhancement was observed, indicating the absence of nonspecific binding. Finally, to exclude the possible effect of components of SSB buffer, we titrated the ssDNA probe with PBS-glycerol, which also did not change the fluorescence (Fig. 5b). These control experiments clearly show that the increase in FMR-DNA fluorescence was induced by its binding to the protein.

To conclude, we have designed, synthesized and characterized new fluorescent $\mathbf{d C}^{\mathbf{X}}$ TPs bearing viscosity-sensitive fluorophores, fluorescent molecular rotors. While the julolidine derivative $\left(\mathbf{d C}^{\mathbf{V}} \mathbf{T P}\right)$ was too bulky for enzymatic incorporation, the smaller $\mathbf{d C}^{\mathbf{V D P}} \mathbf{T P}(\mathbf{4 b})$ was a very good substrate for polymerases in PEX, NEAR and PCR and can be efficiently used in enzymatic construction of DNA probes.
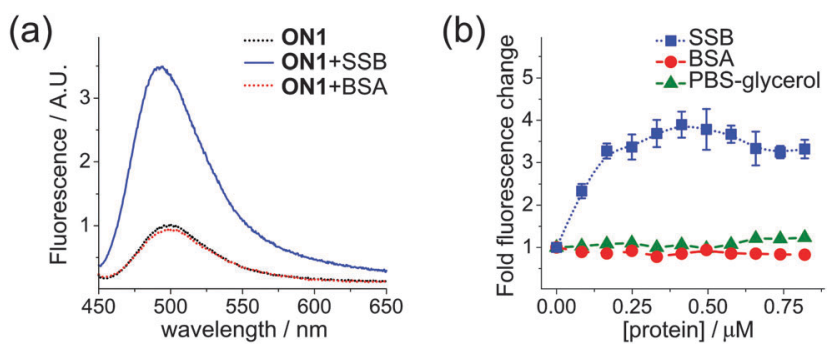

Fig. 5 (a) Fluorescence spectra of the ssDNA probe ON1 (black) in the presence of 0.5 eq. of the SSB tetramer (blue) and BSA (red); (b) relative change in fluorescence of ON1 $(1 \mu \mathrm{M})$ upon addition of the SSB tetramer (blue), BSA (red) and PBS-glycerol (green); titration performed at $\mathrm{pH} 7.4$, $100 \mathrm{mM} \mathrm{NaCl}$; error bars show SD of the mean for $n=3$.
The probe containing modified $\mathbf{d} \mathbf{C}^{\mathbf{V D P}}$ nucleotides exhibits a light-up response upon binding to protein, which makes it prospective for probing DNA-protein interactions under homogenous conditions.

This work was supported by the Academy of Sciences of the Czech Republic (RVO: 61388963), by the Czech Science Foundation (P206-12-G151) and by Gilead Sciences Inc. D.D. thanks the IOCB for postdoctoral fellowship.

\section{Notes and references}

1 (a) N. Dai and E. T. Kool, Chem. Soc. Rev., 2011, 40, 5756-5770; (b) C.-H. Leung, D. S.-H. Chan, H.-Z. He, Z. Cheng, H. Yang and D.-L. Ma, Nucleic Acids Res., 2012, 40, 941-955.

2 (a) M. Hawkins, Cell Biochem. Biophys., 2001, 34, 257-281; (b) A. S. Wahba, A. Esmaeili, M. J. Damha and R. H. E. Hudson, Nucleic Acids Res., 2010, 38, 1048-1056; (c) J.-W. Jung, S. K. Edwards and E. T. Kool, ChemBioChem, 2013, 14, 440-444.

3 T. Ono, S. Wang, C.-K. Koo, L. Engstrom, S. S. David and E. T. Kool, Angew. Chem., Int. Ed., 2012, 51, 1689-1692.

4 J. J. Li, X. Fang, S. M. Schuster and W. Tan, Angew. Chem., Int. Ed., 2000, 39, 1049-1052.

5 (a) S. Park, H. Otomo, L. Zheng and H. Sugiyama, Chem. Commun., 2014, 50, 1573-1575; (b) P. A. Hopkins, R. W. Sinkeldam and Y. Tor, Org. Lett., 2014, 16, 5290-5293; (c) Y. Saito, A. Suzuki, Y. Okada, Y. Yamasaka, N. Nemoto and I. Saito, Chem. Commun., 2013, 49, 5684-5686; (d) D. Dziuba, V. Y. Postupalenko, M. Spadafora, A. S. Klymchenko, V. Guérineau, Y. Mély, R. Benhida and A. Burger, J. Am. Chem. Soc., 2012, 134, 10209-10213; (e) M. Weinberger, F. Berndt, R. Mahrwald, N. P. Ernsting and H.-A. Wagenknecht, J. Org. Chem., 2013，78，2589-2599; $(f)$ T. Kanamori, H. Ohzeki, Y. Masaki, A. Ohkubo, M. Takahashi, K. Tsuda, T. Ito, M. Shirouzu, K. Kuwasako, Y. Muto, M. Sekine and K. Seio, ChemBioChem, 2015, 16, 167-176.

6 (a) M. Hocek and M. Fojta, Chem. Soc. Rev., 2011, 40, 5802-5814; (b) M. Hollenstein, Molecules, 2012, 17, 13569-13591; (c) M. Hocek, J. Org. Chem., 2014, 79, 9914-9921.

7 J. Riedl, R. Pohl, N. P. Ernsting, P. Orsag, M. Fojta and M. Hocek, Chem. Sci., 2012, 3, 2797-2806.

8 J. Riedl, P. Menova, R. Pohl, P. Orsag, M. Fojta and M. Hocek, J. Org. Chem., 2012, 77, 8287-8293.

9 (a) M. A. Haidekker and E. A. Theodorakis, Org. Biomol. Chem., 2007, 5, 1669-1678; (b) M. K. Kuimova, Phys. Chem. Chem. Phys., 2012, 14, 12671-12686.

10 W. L. Goh, M. Y. Lee, T. L. Joseph, S. T. Quah, C. J. Brown, C. Verma, S. Brenner, F. J. Ghadessy and Y. N. Teo, J. Am. Chem. Soc., 2014, 136, 6159-6162.

11 S. Murudkar, A. K. Mora, P. K. Singh and S. Nath, Chem. Commun., 2012, 48, 5301-5303.

12 L. Liu, Y. Shao, J. Peng, C. Huang, H. Liu and L. Zhang, Anal. Chem., 2014, 86, 1622-1631.

13 (a) R. W. Sinkeldam, A. J. Wheat, H. Boyaci and Y. Tor, ChemPhysChem, 2011, 12, 567-570; (b) M. G. Pawar, A. Nuthanakanti and S. G. Srivatsan, Bioconjugate Chem., 2013, 24, 1367-1377; (c) Y. Zhang, X. Yue, B. Kim, S. Yao and K. D. Belfield, Chem. - Eur. J., 2014, 20, 7249-7253; (d) A. A. Tanpure and S. G. Srivatsan, ChemBioChem, 2012, 13, 2392-2399.

14 (a) T. Iwaki, C. Torigoe, M. Noji and M. Nakanishi, Biochemistry, 1993, 32, 7589-7592; (b) Y.-Y. Wu, W.-T. Yu, T.-C. Hou, T.-K. Liu, C.-L. Huang, I. C. Chen and K.-T. Tan, Chem. Commun., 2014, 50, 11507-11510.

15 A. C. Fogarty, E. Duboue-Dijon, F. Sterpone, J. T. Hynes and D. Laage, Chem. Soc. Rev., 2013, 42, 5672-5683.

16 H. Hwang and S. Myong, Chem. Soc. Rev., 2014, 43, 1221-1229.

17 J. Sutharsan, D. Lichlyter, N. E. Wright, M. Dakanali, M. A. Haidekker and E. A. Theodorakis, Tetrahedron, 2010, 66, 2582-2588.

18 J. Ludwig, Acta Biochim. Biophys. Acad. Sci. Hung., 1981, 16, 131-133.

19 (a) V. Raindlová, R. Pohl, M. Šanda and M. Hocek, Angew. Chem., Int. Ed., 2010, 49, 1064-1066; (b) P. Menova and M. Hocek, Chem. Commun., 2012, 48, 6921-6923; (c) P. Ménová, V. Raindlová and M. Hocek, Bioconjugate Chem., 2013, 24, 1081-1093; (d) D. Dziuba, R. Pohl and M. Hocek, Bioconjugate Chem., 2014, 25, 1984-1995.

20 R. D. Shereda, A. G. Kozlov, T. M. Lohman, M. M. Cox and J. L. Keck, Critical Rev. Biochem. Mol. Biol., 2008, 43, 289-318.

21 (a) T. M. Lohman and M. E. Ferrari, Ann. Rev. Biochem., 1994, 63, 527-570; (b) W. Bujalowski and T. M. Lohman, J. Mol. Biol., 1989, 207, 249-268. 